\title{
Task inhibition and task repetition in task switching
}

\author{
Andrea M. Philipp and Iring Koch \\ Max Planck Institute for Human Cognitive and Brain Sciences, Department \\ of Psychology, Munich, Germany
}

\begin{abstract}
In task-switching experiments with three tasks, the relative cost of an $\mathrm{N}-2$ task repetition (task sequence $\mathrm{ABA}$ ) compared to a task switch (task sequence $\mathrm{CBA}$ ) is referred to as $\mathrm{N}-2$ repetition cost. $\mathrm{N}-2$ repetition cost is assumed to reflect persisting inhibition of a task that was recently switched away from. In two experiments, we explored whether the occurrence of task repetitions has an influence on the size of the $\mathrm{N}-2$ repetition cost. The results in both experiments showed a decreased $\mathrm{N}-2$ repetition cost in conditions in which task repetitions were possible - independent of whether the occurrence of task repetitions was manipulated between subjects (Experiment 1) or within subjects (i.e., block by block, Experiment 2). These results suggest that the occurrence of task repetitions affects the balance of activation and inhibition in task switching.
\end{abstract}

The task-switching paradigm offers a tool for studying the role of cognitive control processes that allow a flexible adaptation of internal settings to changing action requirements. Adopting one internal setting always has to go along with the resolution of interference between competing settings. To give a real-life example, when writing an article, the internal setting has to be set on "writing" — which involves the resolution of competition between "writing", "planning the next weekend", and "reading newspapers".

Internal settings are also referred to as "task sets" (see, e.g., Rogers \& Monsell, 1995) and include all relevant components that enable us to perform a particular task (e.g., task relevant stimulus-categorisation rules, category-response rules, corresponding motor programs, etc.). Switching from one task set to another requires the activation of the relevant task set

Correspondence should be addressed to Andrea M. Philipp, RTWH Aachen University, Institute of Psychology, Jägerstr. 17-19, D-52066 Aachen, Germany. E-mail: philipp@psych. rwth-aachen.de

This research was funded by Deutsche Forschungsgemeinschaft Grant KO 2045/4-1 and 4-2 to Iring Koch. We would like to thank Mei-Ching Lien and an anonymous reviewer for comments on a previous version of this paper. We also would like to thank Miriam Gade, Peter Keller, and Stefanie Schuch for helpful discussions concerning this work and Franziska Pöss and Yi Zhang for conducting parts of the experiment. 
and/or the inhibition of irrelevant task sets. Thus, task activation and task inhibition can be considered as cognitive mechanisms for the resolution of interference.

However, these cognitive mechanisms and the process of interference resolution cannot directly be observed in the task-switching paradigm. When using the task-switching paradigm we therefore have to rely on observable phenomena, which are assumed to be empirical markers for cognitive control processes.

Since the first task-switching studies (Jersild, 1927), "N-1 shift cost" is taken as such an empirical marker. In task-switching experiments, the difference in reaction time (RT) and error rate between task-repeat and taskswitch trials represents a disadvantage of switching from one task to another, which is usually denoted as $(\mathrm{N}-1)$ shift cost (e.g., Allport, Styles, \& Hsieh, 1994; Meiran, 1996; Rogers \& Monsell, 1995). It has been suggested that the $\mathrm{N}-1$ shift cost depends largely on the resolution of interference in the preceding trial (e.g., Allport \& Wylie, 2000; Koch \& Philipp, 2005; Schuch \& Koch, 2003). However, currently there is a debate whether N-1 shift cost represents a disadvantage of switching to a task that was inhibited during interference resolution in the preceding trial or an advantage of repeating a task that was activated during interference resolution in the preceding trial (e.g., Dreisbach, Haider, \& Kluwe, 2002; Koch \& Philipp, 2005; Sohn \& Carlson, 2000).

A paradigm has been developed by Mayr and Keele (2000) to show the important role of task inhibition. In the so-called backward inhibition (BI) paradigm, subjects switch among three different tasks. The critical comparison between a task sequence ABA and a task sequence CBA shows that it is more difficult to switch back to a task that has recently been switched away from (i.e., $\mathrm{N}-2$ repetition cost). It has been argued that the engagement in a new task triggers inhibition of the just-executed task, resulting in increased RTs when one has to switch back to this task after only one intermediate trial (e.g., Arbuthnott \& Frank, 2000; Mayr \& Keele, 2000). Like N-1 shift cost, the $\mathrm{N}-2$ repetition cost was supposed to depend to a large degree on interference resolution in the preceding trial (Schuch \& Koch, 2003). However, in contrast to $\mathrm{N}-1$ shift cost, it is widely accepted that $\mathrm{N}-2$ repetition cost results from persisting inhibition of a task that was inhibited during interference resolution in the preceding trial.

In their article, Mayr and Keele (2000) argued that task inhibition is a rather fundamental mechanism because it was also found in a preplanned sequence. That is, even when subjects knew that they would have to switch back to a task after only one intermediate trial they showed substantial N-2 repetition cost. Although keeping the task active in working memory would be quite useful in this context, task inhibition nevertheless seems to have taken place. 
The goal of our study was to examine whether there are variables influencing task inhibition. Specifically, we focused on the question whether the mechanism of task inhibition, and consequently the size of $\mathrm{N}-2$ repetition cost, is affected by the occurrence of task repetitions. Task repetitions might influence the mechanism of task inhibition because in a task repetition the inhibition of the preceding task would be maladaptive, while it would be useful to keep the relevant task set active. To assess whether the occurrence of task repetitions would change the overall amount of task inhibition, we compared task-switching performance in experiments with three tasks, which did or did not include task repetitions. Such a design provides the possibility to study the $\mathrm{N}-2$ repetition cost as difference between an ABA sequence and a control sequence (i.e., CBA). Additionally, $\mathrm{N}-1$ shift cost can be calculated as the difference between a control sequence (i.e., CBA) and a task-repetition sequence (i.e., CAA).

In recent years, several studies have been published in which taskswitching experiments with three different tasks included the occurrence of task repetitions (Arbuthnott \& Frank, 2000; Arbuthnott \& Woodward, 2002; Dreher \& Berman, 2002; Mayr, 2001, 2002; Mayr \& Keele, 2000). To our knowledge, only the study by Mayr and Keele (2000) included experiments with and without task repetitions. But although these authors argued that there is no change in the size of the $\mathrm{N}-2$ repetition cost in an experiment with task repetitions (Experiment 4) as compared to an experiment without task repetitions (Experiment 1), no direct betweenexperiment analysis was reported. However, such a direct comparison would be important because the $\mathrm{N}-2$ repetition cost was not found consistently in all experiments including task repetitions (see, e.g., Arbuthnott \& Woodward, 2002). We intend to focus on this question more systematically by manipulating the occurrence of task repetitions between as well as within subjects.

\section{OVERVIEW OF EXPERIMENTS}

In two experiments, we focused on the question whether the occurrence of task repetitions affects task inhibition and the size of the $\mathrm{N}-2$ repetition cost. Experiment 1 was conducted to study the influence of task repetitions on the $\mathrm{N}-2$ repetition cost between subjects. We compared two experimental groups performing a task-switching experiment with three perceptual decision tasks. For one group the experiment included task repetitions; for the other group there were no task repetitions in the experiment. In Experiment 2, we investigated the role of task repetitions on the $\mathrm{N}-2$ repetition cost within one experiment by alternating between blocks with and without task repetition. 


\section{EXPERIMENT 1}

Subjects in Experiment 1 switched among three perceptual decision tasks. For half of the subjects no repetition of tasks occurred throughout the whole experiment ("no-repetition" group). For the other half of subjects task repetitions could occur ("repetition" group). We expected that the occurrence of task repetitions, in which task inhibition clearly would be maladaptive, might reduce the overall task inhibition level and thus also the $\mathrm{N}-2$ repetition cost.

By introducing task repetitions, we can also compare the size of $\mathrm{N}-2$ repetition cost and $\mathrm{N}-1$ shift cost. We manipulated the duration of the preparation interval (cue-stimulus interval, CSI) to examine whether this manipulation affects $\mathrm{N}-1$ shift cost and $\mathrm{N}-2$ repetition cost differently. We expected to find a reduction of $\mathrm{N}-1$ shift cost with a long preparation time (see, e.g., Meiran, 1996; Rogers \& Monsell, 1995) but no effect of the CSI manipulation on N-2 repetition cost (see, e.g., Hübner, Dreisbach, Haider, \& Kluwe, 2003; Koch, Gade, \& Philipp, 2004; Mayr \& Keele, 2000; Schuch $\&$ Koch, 2003).

\section{Method}

Subjects. Thirty-two subjects ( 19 female and 13 male, mean age $=23.97$ years) were tested and evenly assigned to the two experimental groups "no-repetition" and "repetition". Subjects received $7 €$ for participation.

Stimuli and tasks. Subjects had to switch among three perceptual decision tasks with three-dimensional stimuli. Tasks were to decide about the type (A vs. 4), the size (big vs. small), or the colour (red vs. blue) of a stimulus. Stimuli were presented one at a time in a white frame at the centre of a black screen (15-inch monitor) connected to a PC. The rectangular frame had the width of $4.0 \mathrm{~cm}$ and height of $3.5 \mathrm{~cm}$; the viewing distance was $60 \mathrm{~cm}$. The relevant task in each trial was cued by four signs surrounding the frame (cf. Koch, 2001). The cues were paragraph signs for the type task, up-down pointing arrows for the size task, and small yellow squares for the colour task. Each cue symbol was approximately $0.7 \mathrm{~cm}$ high/wide.

Responses were made manually on an external keyboard with two response keys for the right and left index finger. Response keys measured $1.2 \mathrm{~cm} \times 1.7 \mathrm{~cm}$ and were separated by $3.8 \mathrm{~cm}$.

Procedure. The experiment was run in a single session of approximately 45 minutes. Instructions were given both on the monitor and orally. Instruction emphasised speed as well as accuracy. Subjects were informed about the tasks and the response keys (an instruction sheet concerning the 
mapping of the response keys was in front of them throughout the experiment). All eight possible stimulus-response mappings were counterbalanced across subjects. The instruction explained all three tasks but did not mention the presence (in the repetition group) or absence (no-repetition group) of task repetitions.

Two practice blocks, one with short CSI $(100 \mathrm{~ms})$ and one with long CSI (1000 ms), were run with 12 trials each. The experiment itself consisted of eight blocks of 96 trials. Before each block, subjects were informed about the CSI in the next block. Blocks with short and long CSI alternated; whether the initial CSI was short or long was counterbalanced between subjects. The response-stimulus interval (RSI) was held constant at $1100 \mathrm{~ms}$ with response-cue interval (RCI) being either $100 \mathrm{~ms}$ or $1000 \mathrm{~ms}$, and CSI being either 100 ms or 1000 ms (i.e., RCI 100/CSI 1000 vs. RCI 1000/CSI 100).

In the no-repetition group, the sequence of trials was controlled for an equal number of each task, category and stimulus, response repetition, and all these factors combined. The two relevant task sequences ABA and CBA occurred with a probability $52 \%$ (ABA) or $48 \%$ (CBA) of all trials (excluding the first two trials of each block). Please note that the labels A, B, and C are used as placeholders and stand for each task (i.e., type, size, and colour) with an equal probability. Also, each trial in the experiment was analysed with regard to the two preceding trials as in a "sliding window". Stimuli could be repeated, but not more than once in a row. No task repetition could occur, meaning that subjects had to switch the task in every trial.

For the repetition group, the sequence of trials had a comparable number of each task, ${ }^{1}$ category and stimulus, response repetition, and all these factors combined. The task sequences ABA (30\%), CBA (28\%), and CAA $(26 \%)$ occurred with a similar probability (please note that the sum of probabilities is lower than $100 \%$ because all trials following a task repetition were not analysed). Again, the labels A, B, and C are used as placeholders, which also means that each task (i.e., type, size, and colour) could be repeated. To make task repetitions more salient, a task was sometimes repeated more than once. That is, in the whole experiment there were 11 instances when a task was repeated twice and 3 instances when a task was repeated three times. This way, the overall probability of a task repetition was $27.6 \%$.

A trial started with a black screen followed by the cue (i.e., a white square surrounded by four cue signs). After a variable preparation time (CSI), the stimulus was presented in the middle of the cue frame, simultaneously

\footnotetext{
${ }^{1}$ Due to a programming error the number of tasks differed slightly. That is, the number of colour tasks in each block was reduced by four and in return, the number of type tasks was increased by four. However, the data pattern did not change when we included task as an additional independent variable.
} 
with a tone. Pressing a response key erased the cue and the stimulus from the screen.

Subjects received visual error feedback for $500 \mathrm{~ms}$ when they pressed the wrong key (German: "Falsche Taste").

Design. For both groups, task sequence (ABA vs. CBA in the norepetition group/ABA vs. CBA vs. CAA in the repetition group) and CSI $(100 \mathrm{~ms}$ vs. $1000 \mathrm{~ms})$ were used as within-subject independent variables. Group (no-repetition vs. repetition) was the between-subject variable. With respect to the task-sequence variable, we report two nonorthogonal contrasts. On the one hand, to compare the $\mathrm{N}-2$ repetition cost in both groups we contrasted task sequences $\mathrm{ABA}$ and $\mathrm{CBA}$. On the other hand, to calculate the size of the $\mathrm{N}-1$ shift cost we contrasted task sequences CBA and CAA in the repetition group. RTs and error percentage were measured as dependent variables. Significance was tested at alpha $=.05$.

\section{Results}

The first two trials of each block were discarded from analysis. Only trials preceded by at least two correct trials were included. Additionally, trials with an RT above $3000 \mathrm{~ms}$ were defined as outliers $(0.8 \%$ in both groups). The overall error rate was at $7.4 \%$ in the no-repetition and $7.1 \%$ in the repetition group.

We describe the results in two separate sections. The first section relates to the $\mathrm{N}-2$ repetition cost as a function of the occurrence of task repetitions in the experiment. In the second section we analyse the size of the $\mathrm{N}-1$ shift cost.

$N-2$ repetition cost. We conducted a three-way analysis of variance (ANOVA) on RT data with the within-subject variables task sequence (ABA vs. CBA), and CSI (100 ms vs. $1000 \mathrm{~ms}$ ), and the between-subject variable group (no-repetition vs. repetition). The analysis revealed a significant main effect of task sequence, $F(1,30)=14.2, p=.001$, indicating that RTs for trial $n$ in the task sequence $\mathrm{AB} A(893 \mathrm{~ms})$ were higher than in a task sequence $\mathrm{CB} A$ (859 ms; see Table 1). Importantly, the interaction of task sequence and group was significant as well, $F(1,30)=4.9, p=.034$. The difference between the task sequences $\mathrm{ABA}$ and $\mathrm{CBA}$ was higher in the no-repetition group $(54 \mathrm{~ms})$ than in the repetition group (14 ms). Post hoc $t$-tests for both groups revealed that the $54 \mathrm{~ms}$ effect was significant, $t(1,15)=5.1, p<.001$, whereas the $14 \mathrm{~ms}$ effect was not significant, $t(1,15)=1.0, p=.352$. Thus, the introduction of task repetitions reduced the size of the $\mathrm{N}-2$ repetition cost. 
TABLE 1

Experiment 1: RT in ms (and error percentage) as a function of task sequence (ABA vs. CBA vs. CAA), group (no-repetition vs. repetition), and CSI (100 vs. $1000 \mathrm{~ms}$ )

\begin{tabular}{|c|c|c|c|c|c|}
\hline & \multicolumn{3}{|c|}{ Task sequence } & \multirow{2}{*}{$\begin{array}{c}N-2 \\
\text { repetition cost } \\
(A B A-C B A)\end{array}$} & \multirow{2}{*}{$\begin{array}{c}N-1 \\
\text { shift cost } \\
(C B A-C A A\end{array}$} \\
\hline & $A B A$ & $C B A$ & $C A A$ & & \\
\hline \multicolumn{6}{|c|}{ No-repetition group } \\
\hline CSI 100 & $1004(8.8)$ & $944(8.6)$ & - & $60 *(0.2)$ & - \\
\hline CSI 1000 & $715(7.1)$ & $667(6.2)$ & - & $48 *(0.9)$ & - \\
\hline \multicolumn{6}{|c|}{ Repetition group } \\
\hline CSI 100 & 1078 (7.6) & $1072(7.6)$ & $861(5.7)$ & $6(0.0)$ & $211 *(1.9)$ \\
\hline CSI 1000 & $774(9.6)$ & $752(7.2)$ & $582(4.2)$ & $22(2.4)$ & $170^{*}(3.0)^{*}$ \\
\hline
\end{tabular}

${ }^{*} p<.05(\mathrm{~N}-2$ repetition cost and $\mathrm{N}-1$ shift cost were tested with two-tailed $t$-tests).

$\mathrm{CSI}=$ cue - stimulus interval.

The main effect of CSI was significant, $F(1,30)=100.3, p<.001$, indicating a reduction of RTs with a long CSI (from $1024 \mathrm{~ms}$ to $727 \mathrm{~ms}$ ). However, this effect did not differ between both groups, and did not affect the $\mathrm{N}-2$ repetition cost $(F \mathrm{~s}<1)$. The main effect of group was also not significant $(F<1.4)$.

When conducting the three-way ANOVA on error data, the main effect of task sequence was not significant, $F(1,30)=2.3, p=.140$, but showed the same tendency as in RT data (more errors in an ABA sequence, $8.3 \%$, than in a CBA sequence, $7.4 \%$ ). There also was no difference between groups $(F<1)$. The main effect of CSI was not significant in error data $(F<1.5)$. However, the interaction of CSI and group was significant. The data pattern shows that in the no-repetition group error rate was reduced with long CSI (from $8.7 \%$ to $6.7 \%$ ), whereas there was numerically even a small increase of error rate with long CSI in the repetition group (7.6\% vs. $8.4 \%)$. The interaction of task sequence and CSI was close to significance, $F(1$, $30)=3.1, p=.090$. In tendency, the $\mathrm{N}-2$ repetition cost was increased after a long preparation time (from $0.1 \%$ to $1.7 \%$ ). No other interaction was significant $(F \mathrm{~S}<1)$. As in RT data, the main effect of group was not significant $(F<1)$.

$N-1$ shift cost. We conducted a two-way ANOVA with task sequence (CBA vs. CAA) and CSI (100 ms vs. $1000 \mathrm{~ms})$ as independent variables. Importantly, only data from the repetition group are included in this analysis. The analysis on RT data shows a significant effect of task sequence, $F(1,15)=36.4, p<.001$. RTs in a task repetition sequence CAA were lower (722 ms) than in the control sequence CBA (912 ms), leading to an $\mathrm{N}-1$ shift 
cost of $191 \mathrm{~ms}$. The main effect of CSI was significant, $F(1,15)=66.7$, $p<.001$, but did not significantly interact with task sequence, $F(1,15)=2.8$, $p=.116$. Thus, a long CSI reduced RTs in general (from $967 \mathrm{~ms}$ to $667 \mathrm{~ms}$ ), but the preparatory decrease of $\mathrm{N}-1$ shift cost $(211 \mathrm{~ms} v \mathrm{vs} .170 \mathrm{~ms})$ fell short of significance.

The same analysis on error data only yielded a significant main effect of task sequence, $F(1,15)=10.1, p=.006$. As in RT data, the performance was better in a task sequence CAA (5.0\% errors) than in a CBA sequence $(7.4 \%)$. No other effect or interaction was significant $(F \mathrm{~s}<2.0)$.

\section{Discussion}

The comparison of the size of the $\mathrm{N}-2$ repetition cost between no-repetition and repetition group demonstrated that there is a substantial $\mathrm{N}-2$ repetition cost of $54 \mathrm{~ms}$ in the no-repetition group. In contrast, the repetition group only showed a nonsignificant difference of $14 \mathrm{~ms}$. Thus, including task repetitions in a task-switching experiment with three different tasks reduced the size of the $\mathrm{N}-2$ repetition cost. Importantly, the overall RT level and error rate was not different between both groups, so this difference cannot be accounted for by a change in task difficulty. We therefore assume that the introduction of task repetitions was indeed responsible for the decreased $\mathrm{N}-2$ repetition cost. This result of Experiment 1 is different to what Mayr and Keele (2000) found in their study. We address this difference in the General Discussion.

With regard to preparation time, the data pattern of Experiment 1 is comparable to other studies using three tasks in that the $\mathrm{N}-2$ repetition cost was not influenced by the duration of the preparation interval (Hübner et al., 2003; Koch et al., 2004; Mayr \& Keele, 2000; Schuch \& Koch, 2003). As regards $\mathrm{N}-1$ shift cost, we observed a preparatory decrease of $\mathrm{N}-1$ shift cost of $41 \mathrm{~ms}$. However, in contrast to other task-switching studies with two tasks (e.g., Meiran, 1996; Rogers \& Monsell, 1995), this decrease was not significant $(p=.116)$ in our experiment, which was, as we believe, due to insufficient statistical power.

The major result of Experiment 1 was the between-subject difference of the $\mathrm{N}-2$ repetition cost as a function of the occurrence of task repetitions. To study the influence of task repetitions on the $\mathrm{N}-2$ repetition cost in more detail, we conducted Experiment 2, in which we manipulated the occurrence of task repetitions block by block within subjects. This way, we intended to replicate the finding of Experiment 1 with a different design. We expected to find similar effects when manipulating the occurrence of task repetitions within one experimental group. Additionally, a within-subject manipulation 
is suitable to answer questions about the time course in which the $\mathrm{N}-2$ repetition cost changes as a function of the occurrence of task repetitions.

\section{EXPERIMENT 2}

In Experiment 2, the occurrence of task repetitions was manipulated within subjects by alternating between blocks with and without task repetitions. If the occurrence of task repetitions changes the size of the $\mathrm{N}-2$ task repetition cost, we should find a difference in the $\mathrm{N}-2$ repetition cost between blocks with and blocks without task repetitions.

However, it might be that the size of $\mathrm{N}-2$ repetition cost is not changed from block to block but that the amount of task inhibition adapts to the most suitable compromise to deal with the experimental condition in the course of the experiment. If this were the case, it appears reasonable to assume that at least in the beginning of the experiment, when subjects experience either a block with or without task repetitions, the $\mathrm{N}-2$ repetition cost should be different in both types of blocks. As soon as subjects have experienced both types of blocks (beginning from block 3) the $\mathrm{N}-2$ repetition cost might come to a more intermediate and more constant level. To examine the development of the $\mathrm{N}-2$ repetition cost and $\mathrm{N}-1$ shift cost, we analysed performance as a function of "experience" (block $1 / 2$ vs. $3 / 4$ vs. $5 / 6$ vs. $7 / 8)$.

In Experiment 1, as in other studies (Hübner et al., 2003; Koch et al., 2004; Mayr \& Keele, 2000; Schuch \& Koch, 2003), the N-2 repetition cost was not influenced by preparation time. Therefore, we saw no important theoretical implication to manipulate the CSI, so that we kept the CSI constant at $100 \mathrm{~ms}$ in Experiment 2.

\section{Method}

Subjects. Thirty-two new subjects $(25$ female and 7 male, mean age $=$ 23.2 years) were tested and received $7 €$.

Stimuli, tasks, procedure, and design. The experimental setting was comparable to Experiment 1. The experiment contained 8 blocks of 96 trials each and lasted about 45 minutes. However, in Experiment 2 subjects alternated between blocks with and without task repetitions. For half the subjects the experiment started with a block including task repetitions, for the other half the first block contained no task repetitions. Before each block subjects were told whether or not they should expect task repetitions in the next block. Thus, in contrast to Experiment 1, in the beginning of the experiment we mentioned the possible occurrence of task repetitions. As this makes task repetitions already quite salient, we decided not to repeat a task 
more than once in Experiment 2. We controlled the overall trial sequence in order to ensure a similar number of each relevant task sequence (ABA vs. CBA in no-repetition blocks and ABA vs. CBA vs. CAA in repetition blocks). In no-repetition blocks $55 \%$ of all trials (without the first two trials) were $\mathrm{ABA}$ trials, and $45 \%$ were $\mathrm{CBA}$ trials. In repetition blocks, $29 \%$ were ABA trials, $29 \%$ were CBA trials, and $28 \%$ were CAA trials (again the sum is less than $100 \%$ because all trials following a task repetition were not analysed). Thus, the probability of a task repetition in repetition blocks was about the same as in the repetition group of Experiment 1.

For RT analysis, task sequence (ABA vs. CBA vs. CAA), type of block (no-repetition vs. repetition), and number of block ( $1 / 2$ vs. $3 / 4$ vs. $5 / 6$ vs. $7 / 8$ ) were used as within-subject independent variables. With respect to the tasksequence variable, again we report two nonorthogonal contrasts. In one contrast we report the comparison of the $\mathrm{N}-2$ repetition cost in blocks with and without task repetitions (task sequences $\mathrm{ABA}$ vs. CBA); in the other contrast we report the size of the $\mathrm{N}-1$ shift cost (task sequences CBA and CAA) in blocks with task repetition. As number of block is a variable with four levels, we will report $\varepsilon$-values when different from 1.0 and use the Huynh-Feldt test to report $p$ values based on corrected degrees of freedom. However, we still report noncorrected degrees of freedom.

Including number of block as independent variable reduces the number of observations for each cell. Because this, above all, affects the statistical power of the error analysis, we collapsed error data across blocks. That is, for the error analysis only task sequence (ABA vs. CBA vs. CAA) and type of block (no-repetition vs. repetition) were used as within-subject independent variables. However, with respect to the variable task sequence we report the same contrasts as mentioned for the RT analysis.

\section{Results}

The first two trials of each block were discarded from analysis as well as trials with an RT above $3000 \mathrm{~ms}$ ( $1.7 \%$ of otherwise correct trials). Only trials preceded by at least two correct trials were included. The overall error rate was $7.1 \%$.

$N-2$ repetition cost. A three-way ANOVA on $\mathrm{RT}$ data with the independent variables task sequence (ABA vs. CBA), type of block (norepetition vs. repetition), and number of block ( $1 / 2$ vs. $3 / 4$ vs. $5 / 6$ vs. $7 / 8$ ) revealed significant main effects of number of block, $F(3,93)=164.6, p<.001$, $\varepsilon=0.595$, and task sequence, $F(1,31)=66.5, p<.001$. RTs decreased in the course of the experiment (from $1352 \mathrm{~ms}$ in block $1 / 2$ to $904 \mathrm{~ms}$ in block 7/8), and RTs were higher in a task sequence ABA (1103 ms) than in a task 
sequence CBA (1046 ms). The main effect of type of block was not significant $(F<1)$. Importantly, the interaction of task sequence and type of block was significant, $F(1,31)=6.8, p=.014$. That is, the $\mathrm{N}-2$ repetition cost significantly differed between blocks with task repetitions $(46 \mathrm{~ms})$ and blocks without task repetitions $(70 \mathrm{~ms})$. The interaction of task sequence, type of block, and number of block was significant as well, $F(3,93)=4.9$, $p=.003, \varepsilon=0.985$. In separate analyses for each pair of blocks, the interaction between task sequence and type of block was significant only for block $1 / 2, F(1,31)=13.3, p=.001$, showing a substantial $\mathrm{N}-2$ repetition cost of $87 \mathrm{~ms}$ in blocks without task repetitions and an N-2 repetition benefit of $16 \mathrm{~ms}$ in blocks with task repetitions (see Figure 1). In all other blocks this interaction was not significant $(F \mathrm{~s}<1.4)$. The data pattern for theses blocks show $\mathrm{N}-2$ repetition cost in blocks with and without task repetitions. (For error data, the two-way ANOVA with task sequence and type of block revealed no significant effects; $F \mathrm{~s}<1$.)

$N-1$ shift cost. We conducted a two-way ANOVA with task sequence (CBA vs. CAA) and number of block. Please note that only data from repetition blocks are included in this analysis. The analysis yielded significant main effects of number of block, $F(3,93)=87.9, p<.001, \varepsilon=0.715$, and task sequence, $F(1,31)=36.6, p<.001$. RTs decreased over the experiment (from $1241 \mathrm{~ms}$ to $828 \mathrm{~ms}$ ) and were lower in task repetitions (924 ms) than in task switches $(1053 \mathrm{~ms})$. Additionally, the interaction of task sequence and number of block was significant, $F(3,93)=6.3, p=.003, \varepsilon=0.673$. The data pattern indicates that an $\mathrm{N}-1$ shift cost was found in all blocks but that

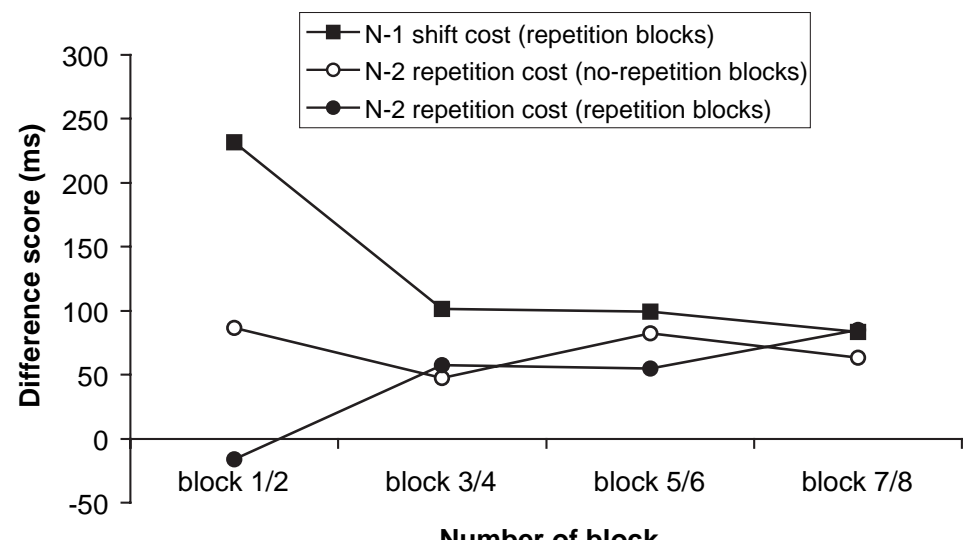

Number of block

Figure 1. Size of $\mathrm{N}-1$ shift cost and $\mathrm{N}-2$ repetition cost (in ms) as a function of block (1/2 vs. $3 / 4$ vs. $5 / 6$ vs. $7 / 8$ ) and type of block (no-repetition vs. repetition) in Experiment 2. 
it was substantially larger in block $1 / 2(232 \mathrm{~ms})$ than in the other blocks (101 ms in block 3/4, $100 \mathrm{~ms}$ in block 5/6, $83 \mathrm{~ms}$ in block 7/8).

With regard to error data, a paired-samples $t$-test was calculated to compare task sequences CBA and CAA in repetition blocks. The two-tailed $t$-test was significant, $t(1,31)=2.1, p=.037$, showing less errors in a task repetition $(5.7 \%)$ than in a task switch $(7.3 \%)$.

\section{Discussion}

Experiment 2 replicated the finding that the size of the $\mathrm{N}-2$ repetition cost is dependent on the experimental condition. In the first block without task repetitions, subjects showed a large $\mathrm{N}-2$ repetition cost $(87 \mathrm{~ms})$, whereas the data pattern was even slightly reversed ( $\mathrm{N}-2$ repetition benefit of $16 \mathrm{~ms})$ in blocks with task repetitions. Additionally, the $\mathrm{N}-1$ shift cost was extremely large $(232 \mathrm{~ms})$ in the first block with task repetitions. This data pattern is consistent with the idea that in the first block without task repetitions task inhibition was resulting in a substantial $\mathrm{N}-2$ repetition cost. However, in the first block with task repetitions task inhibition is rather maladaptive to resolve the interference between competing tasks. The large benefit of repeating a task and the absence of an $\mathrm{N}-2$ repetition benefit may imply that task activation played a larger role in the first block with task repetitions. The alternation between blocks with and without task repetitions did not change the $\mathrm{N}-2$ repetition cost from block to block, but might have led to an intermediate level of both task activation and task inhibition.

\section{GENERAL DISCUSSION}

The present experiments examined the relation of task repetitions and the $\mathrm{N}-2$ repetition cost. In both experiments the $\mathrm{N}-2$ repetition cost was influenced by the occurrence or nonoccurence of task repetitions. In conditions in which task repetitions were possible we found a decreased $\mathrm{N}-2$ repetition cost (Experiment 1, block 1/2 of Experiment 2).

We are aware of six other studies in which task repetitions occurred in experiments with three tasks, and which thus are relevant to explore the influence of task repetitions on $\mathrm{N}-2$ repetition cost (Arbuthnott \& Frank, 2000; Arbuthnott \& Woodward, 2002; Dreher \& Berman, 2002; Mayr, 2001, 2002; Mayr \& Keele, 2000). Three of these studies (Mayr, 2001, 2002; Mayr \& Keele, 2000) report finding $\mathrm{N}-1$ shift cost and $\mathrm{N}-2$ repetition cost. Arbuthnott and Frank (2000) report an N-2 repetition cost but no significant $\mathrm{N}-1$ shift cost. In the experiment conducted by Dreher and Berman (2002), the $\mathrm{N}-2$ repetition cost was found, but a possible $\mathrm{N}-1$ shift cost was not reported. Finally, the study of Arbuthnott and Woodward 
(2002) demonstrated robust $\mathrm{N}-1$ shift cost under all conditions, whereas the $\mathrm{N}-2$ repetition cost occurred only under some conditions. Taken together, it seems that there is no consistent data pattern with respect to the occurrence of $\mathrm{N}-1$ shift cost and $\mathrm{N}-2$ repetition cost. Our experiments indicated that task repetitions influence the size of $\mathrm{N}-2$ repetition cost.

To speculate about the possible effect of task repetitions, it can be stated that the inhibition of the previous task is not useful in task repetitions. Rather, persistence of task activation is necessary to respond according to the correct task in a task repetition. Thus, the question can be raised whether this emphasis on task activation in some way influenced the task inhibition that presumably underlies the occurrence of $\mathrm{N}-2$ repetition cost (cf. Mayr \& Keele, 2000). However, suggesting that the occurrence of task repetitions had a different influence on the amount of task activation (i.e., increase) and task inhibition (i.e., reduction) appears to challenge theoretical notions that assume a direct functional link between task activation and task inhibition - for example "lateral inhibition".

Lateral inhibition was assumed to be a likely mechanism in cognitive control (see, e.g., Norman \& Shallice, 1986) and in particular in task switching (see, e.g., Mayr \& Keele, 2000). The idea of lateral inhibition in task switching suggests that task inhibition is a direct byproduct of task activation, so that there is a direct functional link between activation and inhibition. In other words, the stronger the activation of the relevant task is, the stronger is the concomitant inhibition of irrelevant task(s). This assumption, however, is in discordance with our observations because according to the lateral inhibition view the occurrence of task repetitions (and thus presumably of strong task activation) should have resulted in an even increased $\mathrm{N}-2$ repetition cost.

A critical test for a direct functional link between task activation and task inhibition is to examine their temporal dynamics. As regards task activation, one could argue that task activation starts as soon as a cue was presented. This assumption is supported by the finding that the $\mathrm{N}-1$ shift cost depends on the length of the CSI (see, e.g., Meiran, 1996). In contrast, the N-2 repetition cost is not influenced by CSI duration (e.g., Mayr \& Keele, 2000). Thus, it is unlikely that task inhibition is triggered by the cue. Yet, there still remain two possibilities when task inhibition might take place: The occurrence of task inhibition could depend on a stimulus-based conflict between tasks, or task inhibition could take place after the execution of a response as "self-inhibition" of the just executed task. Note that in the first possibility the inhibition affects tasks that are irrelevant in the present trial (i.e., competing tasks), whereas the self-inhibition would affect the just executed task. It was shown elsewhere that the self-inhibition of a task is unlikely (Hübner et al., 2003; Mayr \& Keele, 2000; Schuch \& Koch, 2003). Therefore, task inhibition seems to depend on the need to resolve 
interference between tasks and that this interference resolution takes place after the presentation of a stimulus (cf. Schuch \& Koch, 2003). Thus, task activation and task inhibition appear to have different temporal loci.

Yet, although the temporal characteristics of task activation and task inhibition appear to be different, we nevertheless suggest an indirect functional link between task activation and task inhibition. It was supposed that the persisting activation of the preceding trial causes interference in the actual trial (see, e.g., Koch \& Philipp, 2005). The amount of task inhibition that is necessary to resolve this proactive interference in the actual trial thus depends on the amount of persisting activation (cf. Gade \& Koch, 2005). Therefore, on a trial-by-trial basis there might be an indirect functional link between task activation and task inhibition.

However, this indirect functional link on a trial-by-trial basis cannot account for the general reduction of the $\mathrm{N}-2$ repetition cost we observed in experiments with task repetitions. We speculate that although task activation and task inhibition are indirectly functionally linked on a trial-by-trial basis, they are also (at least partially) dissociable on a more general level. We suggest that task repetitions change the general process of how to resolve interference. Task activation (i.e., activating the relevant task) and task inhibition (i.e., inhibiting irrelevant tasks) are two mechanisms of interference resolution and we assume that both mechanisms are integrated in the general process of interference resolution. The resolution of interference might be possible by different processes: by the activation of the relevant task alone, by the activation of the relevant task and the inhibition of irrelevant tasks, and by the inhibition of irrelevant tasks alone. We believe that interference resolution is due to task activation and task inhibition. However, the ratio of task activation and task inhibition in the process of interference resolution might be variable. It seems that in an experimental condition in which strong task activation is helpful (i.e., when task repetitions are possible), the relative amount of task inhibition is reduced, and vice versa (see also Arbuthnott \& Frank, 2000).

The assumption that the interplay of task activation and task inhibition is to some point variable appears to be supported by a dissociation of the functional role of the right and left prefrontal cortex. The prefrontal cortex (PFC) is assumed to play a role for switching between tasks and for the resolution of interference (see e.g., Miller \& Cohen, 2001). However, different areas in the PFC can be functionally dissociated. Whereas areas of the right PFC seems to play a major role in the inhibition of irrelevant action rules and task sets (for a review, see Aron, Robbins, \& Poldrack, 2004), the left PFC seems to be more important in maintaining a task set (MacDonald, Cohen, Stenger, \& Carter, 2000). As regards the role of these cortical areas for task switching, it was demonstrated that patients with right and left frontal lesions both show impaired performance but that the origin for their impairment 
appears to be different (Aron, Monsell, Sahakian, \& Robbins, 2004). Right frontal patients were more impaired in inhibiting irrelevant responses, whereas left frontal patients showed a pattern of results that might be interpreted as a lack of task maintenance. This neuropsychological dissociation is consistent with our speculation that the ratio of task activation and task inhibition in the process of interference resolution is variable.

Importantly, this speculation refers to the general "setting" of which process is used to resolve the interference within one experiment and not to the amount of task activation and task inhibition that is needed to resolve the interference in each single trial. Experiment 2 has shown that the $\mathrm{N}-2$ repetition cost was on an intermediate level after participants had experienced repetition and no-repetition blocks. Therefore, it is unlikely that the reduction of the $\mathrm{N}-2$ repetition cost we observed depends on a factor that varies on a block-by-block or even trial-by-trial basis. Rather, the occurrence of task repetitions seems to influence the general process of interference resolution. Consequently, the general "setting" of how much task activation and task inhibition is used to resolve interference cannot explain trial by trial variations of task activation and task inhibition that lead, for example, to the occurrence of asymmetrical shift cost (cf. Allport et al., 1994). Still, further research will have to reconcile the proposed partial dissociability of task activation and task inhibition in the general setting of interference resolution and the indirect functional link between task activation and task inhibition on a trial-by-trial basis.

To summarise, we have shown that under experimental conditions in which task repetitions are possible, the $\mathrm{N}-2$ repetition cost is decreased and therefore harder to find as empirical marker for effects of interference resolution. The results of our experiments thus indicate that sequential task control is not a fixed but rather a flexible mechanism consisting of different components (i.e., task activation and task inhibition).

PrEview proof published online 24 February 2006

\section{REFERENCES}

Allport, A., \& Wylie, G. (2000). Task-switching, stimulus-response bindings, and negative priming. In S. Monsell \& J. S. Driver (Eds.), Attention and performance XVIII: Control of cognitive performance (pp. 35-70). Cambridge, MA: MIT Press.

Allport, D. A., Styles, E. A., \& Hsieh, S. (1994). Shifting intentional set: Exploring the dynamic control of tasks. In C. Umiltà \& M. Moscovitch (Eds.), Attention and performance $X V$ : Conscious and nonconscious information processing (pp. 421-452). Cambridge, MA: MIT Press.

Arbuthnott, K., \& Frank, J. (2000). Executive control in set switching: Residual switch cost and task-set inhibition. Canadian Journal of Experimental Psychology, 54, 33-41. 
Arbuthnott, K. D., \& Woodward, T. S. (2002). The influence of cue-task association and location on switch cost and alternating-switch cost. Canadian Journal of Experimental Psychology, 56, $18-29$.

Aron, A. R., Monsell, S., Sahakian, B. J., \& Robbins, T. W. (2004). A componential analysis of task-switching deficits associated with lesions of left and right frontal cortex. Brain, 127, 1561-1573.

Aron, A. R., Robbins, T. W., \& Poldrack, R. A. (2004). Inhibition and the right inferior frontal cortex. Trends in Cognitive Sciences, 8, 170-177.

Dreher, J. C., \& Berman, K. F. (2002). Fractionating the neural substrate of cognitive control processes. Proceedings of the National Academy of Science of the USA, 99, 14595-14600.

Dreisbach, G., Haider, H., \& Kluwe, R. H. (2002). Preparatory processes in the task-switching paradigm: Evidence from the use of probability cues. Journal of Experimental Psychology: Learning, Memory, and Cognition, 28, 468-483.

Gade, M., \& Koch, I. (2005). Linking inhibition to activation in the control of task sequences. Psychonomic Bulletin and Review, 12, 530-534.

Hübner, M., Dreisbach, G., Haider, H., \& Kluwe, R. H. (2003). Backward inhibition as a means of sequential task-set control: Evidence for reduction of task competition. Journal of Experimental Psychology: Learning, Memory, and Cognition, 29, 289-297.

Jersild, A. T. (1927). Mental set and shift. Archives of Psychology (Whole No. 89).

Koch, I. (2001). Automatic and intentional activation of task sets. Journal of Experimental Psychology: Learning, Memory, and Cognition, 27, 1474-1486.

Koch, I., Gade, M., \& Philipp, A. M. (2004). Inhibition of response mode in task switching. Experimental Psychology, 51, 51-57.

Koch, I., \& Philipp, A. M. (2005). Effects of response selection on the task-repetition benefit in task switching. Memory and Cognition, 33, 624-634.

MacDonald, A. W., Cohen, J. D., Stenger, V. A., \& Carter, C. S. (2000). Dissociating the role of the dorsolateral prefrontal and anterior cingulated cortex in cognitive control. Science, 288, 1835-1838.

Mayr, U. (2001). Age differences in the selection of mental sets: The role of inhibition, stimulus ambiguity, and response-set overlap. Psychology and Aging, 16, 96-109.

Mayr, U. (2002). Inhibition of action rules. Psychonomic Bulletin and Review, 9, 93-99.

Mayr, U., \& Keele, S. W. (2000). Changing internal constraints on action: The role of backward inhibition. Journal of Experimental Psychology: General, 129, 4-26.

Meiran, N. (1996). Reconfiguration of processing mode prior to task performance. Journal of Experimental Psychology: Learning, Memory, and Cognition, 22, 1423-1442.

Miller, E. K., \& Cohen, J. D. (2001). An integrative theory of prefrontal cortex function. Annual Review of Neuroscience, 24, 167-202.

Norman, D. A., \& Shallice, T. (1986). Attention to action: Willed and automatic control of behaviour. In R. J. Davidson, G. E. Schwartz, \& D. Shapiro (Eds.), Consciousness and self-regulation (Vol. 4, pp. 1-18). New York: Plenum.

Rogers, R. D., \& Monsell, S. (1995). Costs of a predictable switch between simple cognitive tasks. Journal of Experimental Psychology: General, 124, 207-231.

Schuch, S., \& Koch, I. (2003). The role of response selection for inhibition of task sets in task shifting. Journal of Experimental Psychology: Human Perception and Performance, 29, 92-105.

Sohn, M.-H., \& Carlson, R. A. (2000). Effects of repetition and foreknowledge in task-set reconfiguration. Journal of Experimental Psychology: Learning, Memory, and Cognition, 26, 1445-1460. 